\title{
Analysis of regional left ventricular wall movement by phased array echocardiography
}

\author{
D. G. GIBSON, D. J. BROWN, AND R. B. LOGAN-SINCLAIR \\ From Brompton Hospital, London
}

SUMMARY Images from standard two-dimensional echocardiographs do not lend themselves to the study of regional left ventricular wall movement because of poor definition of endocardium. An alternative method, based on a wide-angle phased array sector scanner has, therefore, been devised. Once the appropriate region of the heart has been identified, the scan rate is increased, and an array of $41 \mathrm{M}$ mode scans is produced, resulting in improved frequency response and endocardial definition. Each scan is digitised separately, and since their spatial orientation is known, the left ventricular image can be reconstituted by computer, and isometric or contour displays produced. Preliminary results, based on 40 patients, are presented showing examples of left ventricular wall movement. Regional abnormalities of amplitude or timing can be displayed with results similar to those of angiography, while disturbances of septal motion or wall thickness can be observed in greater detail than with other methods.

A major application of $M$-mode echocardiography has been in the study of left ventricular function. However, this method is not capable of giving direct information about the distribution of abnormally contracting segments when the pattern of wall movement is non-uniform. For this reason, the potential of two-dimensional echocardiographic systems, both phased array (Kisslo et al., 1977) and mechanical (Weyman et al., 1976), has been investigated in patients with coronary artery disease in order to detect such disturbances. These studies have been limited, however, because of the purely subjective method of analysis of the echocardiograms that was used. We have, therefore, developed a method of display of regional left ventricular wall movement which not only allows abnormalities of amplitude and direction, but also of timing to be documented objectively. Preliminary results in an illustrative series of patients are presented here.

\section{Methods}

Echocardiographic images of the left ventricular cavity in long axis view were recorded in 40 patients, using a prototype phased array system, scanning a sector of 85 degrees with 246 lines per sector and 8 sector scans per second. Each frame was stored on a standard video casette tape-recorder. When

Received for publication 10 July 1978 the appropriate landmarks: the aortic root, mitral valve, septal and posterior wall endocardium, and apex had been identified, the number of scan lines was reduced from 246 to 41 , and the scan rate correspondingly increased to 50 per second, for a period of 3 seconds. These frames were also stored on video tape and then replayed, not as a twodimensional image, but as a series of $41 \mathrm{M}$-mode echocardiograms on to a Honeywell 1851 stripchart recorder, operating at a paper speed of $50 \mathrm{~mm} / \mathrm{s}$. The resulting $41 \mathrm{M}$-mode echocardiograms had a pulse frequency of $50 \mathrm{~Hz}$, and scanned the left ventricle from apex to aortic root in long axis view for the same period of 3 seconds in each. The electrocardiogram was made to modulate a $3 \mathrm{KHz}$ carrier, and this was recorded on the audio channel of the tape-recorder. A representative example is shown in Fig. 1.

\section{DIGITISING METHOD}

The $41 \mathrm{M}$-mode echocardiograms were digitised manually using a Summagraphics digitiser and Prime 300 computer system, in a similar way to that used for single $\mathrm{M}$-mode echograms (Gibson and Brown, 1973). For each M-mode, calibration points corresponding to time, depth, and the $\mathrm{Q}$ waves bounding the beat to be studied were digitised. Right and left septal echoes, those from anterior and posterior cusps or papillary muscles of the mitral valve, and those corresponding to endo- and epicardial surfaces of the posterior wall, 


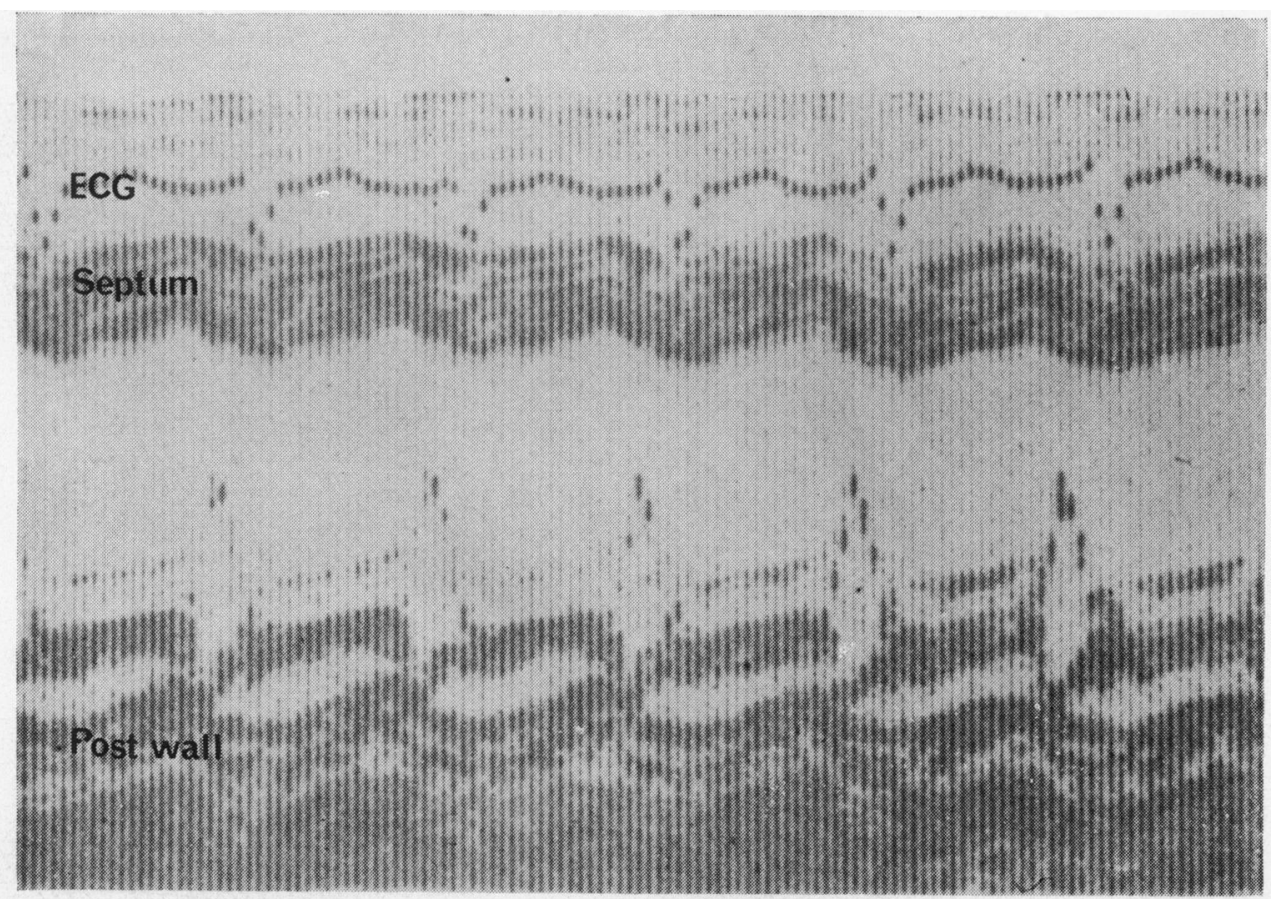

Fig. 1 Single $M$-mode recording at the level of the mitral valve from a normal subject, showing, in addition, right and left septal echoes, and those from the posterior wall and posterior chorda tendineae.

were then digitised, and a series of linearly interpolated points generated along each trace at intervals of $20 \mathrm{~ms}$. If any structure was absent or inadequately defined, such as the mitral valve apparatus below the insertion of the papillary muscles or the endocardium towards the apex, then there was no necessity to digitise the corresponding echo on that particular M-mode.

This digitising technique, together with information about scan depth (derived from scan time and the velocity of sound) and the angle between scan lines, resulted in a matrix of $41 \mathrm{M}$-mode echoes from the 6 structures, with a known time and spatial relation to each other, so that the position in space of the original echoes could be reconstructed.

\section{DISPLAY METHODS}

The information was displayed in a number of ways:

(1) Digitised data from a single $\mathbf{M}$-mode could be plotted unchanged. This was of value in identifying the time of events such as mitral valve opening, which could then be superimposed on all other records.

(2) The position of structures in space at any time, to the nearest $20 \mathrm{~ms}$ after the $Q$ wave, could be displayed as shown in Fig. 2. This could be regarded as analogous to a single cineangio frame.
MULTIPLE M-MODE TECHNIQUE

SINGLE FRAME

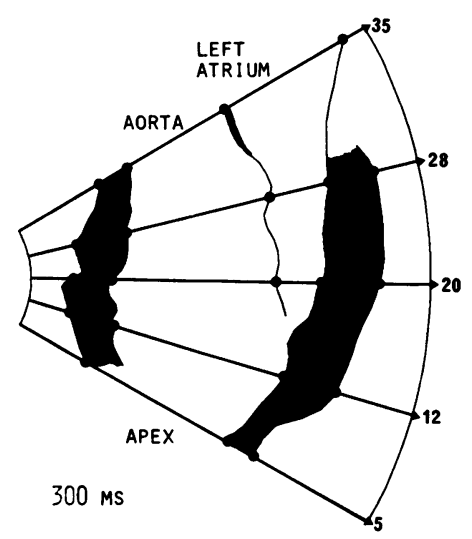

Fig. 2 Single frame display, showing position of septum, posterior wall, and mitral valve $300 \mathrm{~ms}$ after the $Q R S$ onset in a patient with a recent myocardial infarction.

(3) Endocardial echoes for any predetermined period of the cardiac cycle could be plotted superimposed to give an impression of regional amplitude of movement (Fig. 3).

(4) Plots from each $\mathrm{M}$-mode of a single structure, 


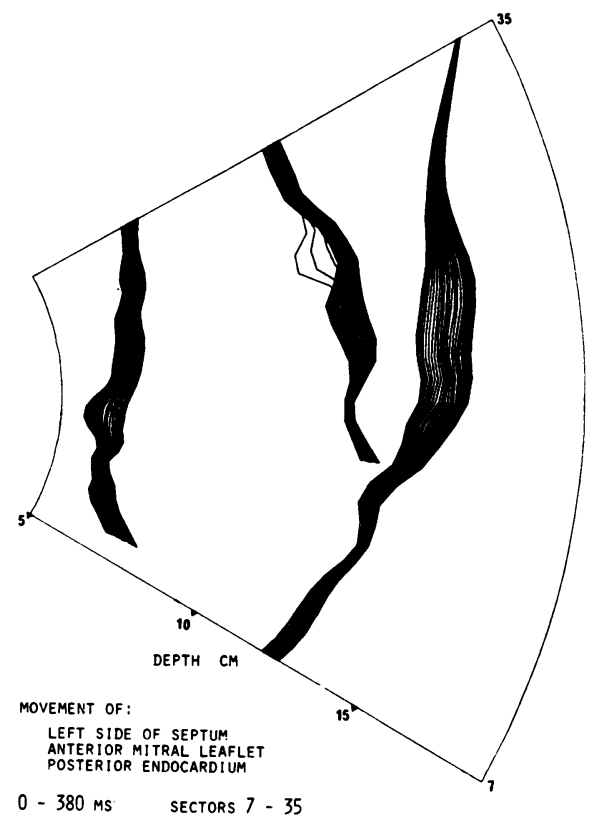

Fig. 3 Superimposed outlines of left septal and posterior wall endocardium and anterior cusp echoes from the same patient as Fig. 2, during systole (0-380 ms after the onset of the QRS complex). There is abnormal movement of the septum, and of the posterior wall below the level of the insertion of the papillary muscle.

such as the left side of the septum or the endocardial surface of the posterior wall could be stacked obliquely, with a series of isochrones superimposed joining events occurring simultaneously. The resulting display produced a three-dimensional effect which allowed regional amplitude and timing of movement to be appreciated. Such plots could be produced either for the position of individual structures relative to the transducer on the chest wall, or their position at end-diastole, or for the distance between two structures such as wall thickness measured as the distance between endoand epicardial surfaces of the posterior wall. As an alternative to the isometric display, the same information could be presented using a set of contour lines (Gibson et al., 1976).

\section{Results}

Typical results are shown in Fig. 2 to 7 . Fig. 2 shows the position of the right and left sides of the septum, anterior mitral valve cusp, and endoand epicardial surfaces of the posterior wall $300 \mathrm{~ms}$ after the $Q$ wave. Superimposed outlines of the left septal and posterior wall endocardium are (a)

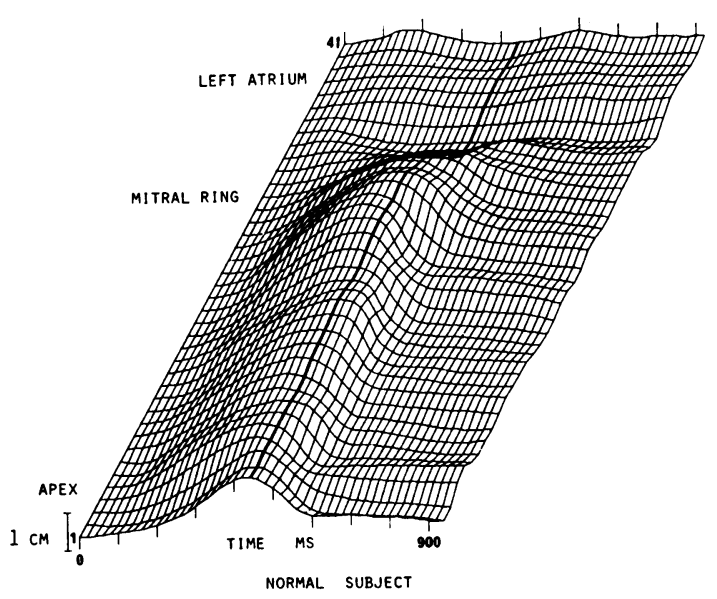

(b)

POSTERIOR ENDOCARDIAL POSITION

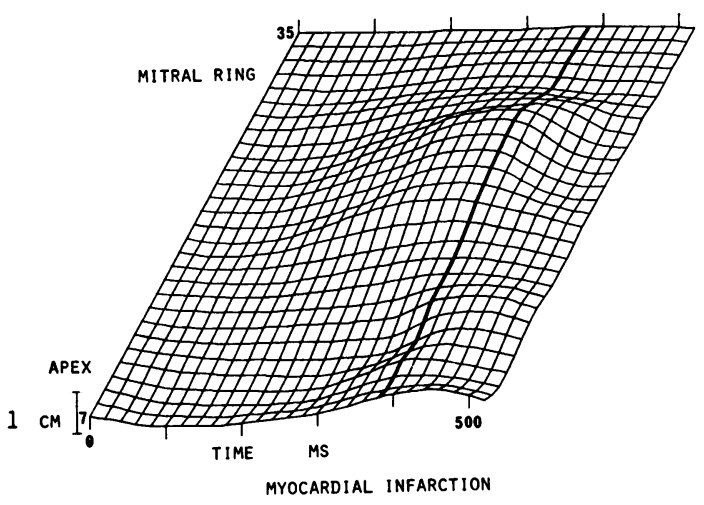

Fig. 4 (a) Isometric display of posterior endocardial movement from a normal subject, from the apex of the left ventricle to the level of mid-left atrium. The accentuated isochrone represents the timing of mitral valve opening. The synchronous pattern of endocardial movement is apparent. (b) Isometric display of posterior endocardial movement from the patient illustrated in Fig. 2 and 3. There is abnormal bulging of the ventricle during systole, with delayed inward movement after mitral valve opening.

shown in Fig. 3. It appears that the amplitude of wall movement is normal in the posterior wall at the level of the mitral valve, but below this its amplitude is reduced and time relations are unclear because the lines are crossing. Similarly, the pattern of septal movement is indeterminate; indeed it is not even possible to tell whether the dominant movement is normal or reversed, indicating the 
limitations of this method of display which essentially reconstructs the original cavity outlines to delineate regional abnormalities of movement. In contrast, isometric displays from this same subject (Fig. 4b) and a normal subject (Fig. 4a) are shown for comparison. In the normal subject, the co-ordinate pattern of left ventricular wall movement from the apex to the mitral ring is readily apparent. In Fig. 4a, however, there is normal movement in the upper part of the cavity, but outward movement during systole and delayed inward movement during diastole towards the apex. In Fig. 5 are shown similar plots of septal movement from a normal subject (Fig. 5a), a patient with a mitral Starr-Edwards prosthesis (Fig. 5c), where movement is reversed, and from a patient with an ostium secundum atrial septal defect (Fig. 5b), where it is normal towards the apex, and reversed towards the aortic root, the cross-over point being readily apparent (Hagan et al., 1974). In Fig. 6a and 6b are shown the plots of posterior wall thickness corresponding to Fig. $4 \mathrm{a}$ and $4 \mathrm{~b}$.

\section{Discussion}

Two-dimensional echocardiographic techniques have great potential in studying left ventricular function. However, initial attempts at digitising endocardial echoes directly from stop frames have, in our hands, been disappointing. Though the cavity outlines were clearly apparent on the moving image, no sooner was it stopped than all semblance of continuity disappeared. The present method was therefore devised in order to overcome this difficulty, and appeared capable of showing endocardial echoes,

(a)

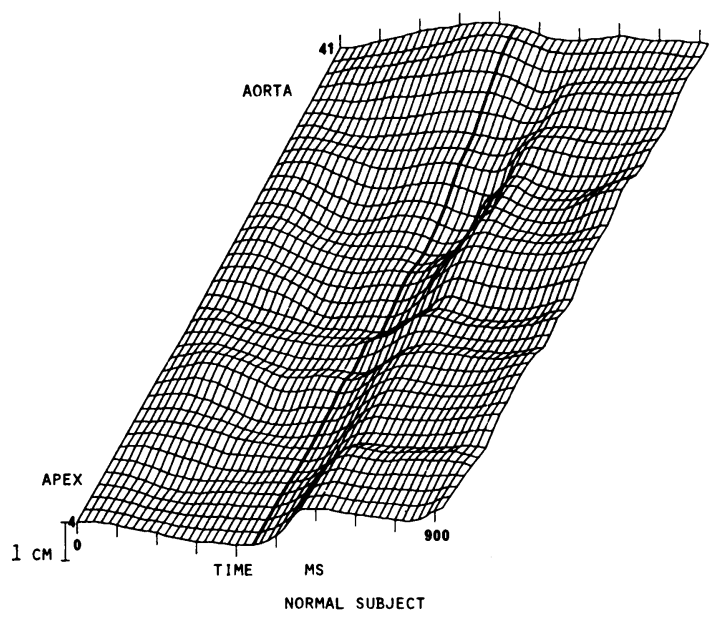

(b)

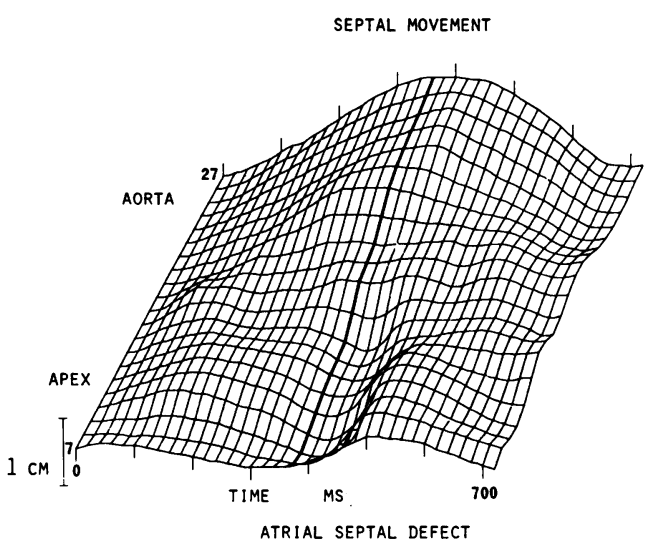

(c)

SEPTAL MOVEMENT

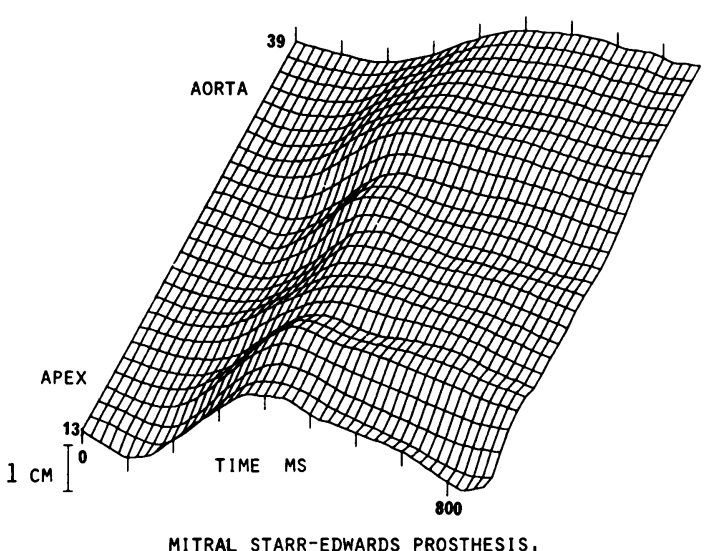

Fig. 5 (a) Isometric display of the normal pattern of septal movement from apex to aortic root, in a normal subject. The accentuated isochrone represents the timing of mitral valve opening. (b) $A$ similar plot from a patient with an atrial septal defect showing reversed upper septal movement. (c) $A$ similar plot from a patient with a mitral Starr-Edwards prosthesis, showing reversed septal movement of all parts of the septum accessible to study.

of a quality which could easily be digitised manually, from part of the cavity in approximately 50 per cent of patients studied. In the form in which it was implemented, the additional cost to the scanner was only some 5 per cent of a video disc system. It had the limitation that the lines along which the image could be examined were only those from which it was constructed. It was thus most effective when the surfaces to be studied were perpendicular to the scan lines, and least so when they were parallel, making it unsuitable for analysis of short axis views. This has been achieved by J. Garrison 
(a)

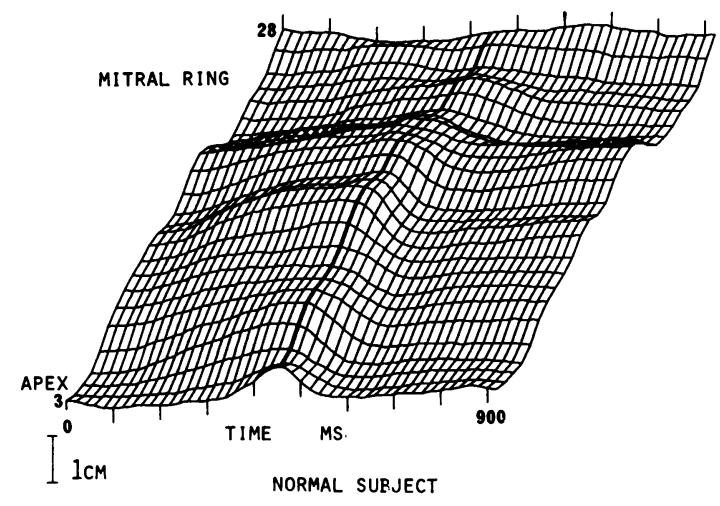

(b)

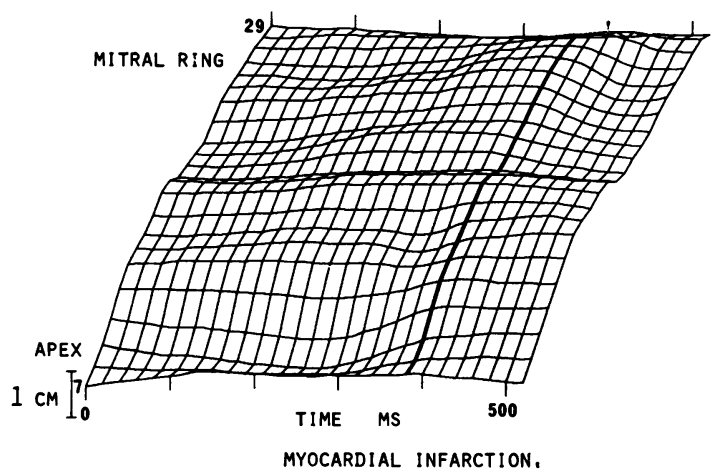

Fig. 6 (a) Display of posterior wall thickness from a normal subject, showing an increase during systole and rapid thinning early in diastole, after mitral valve opening. (b) Posterior wall thickness changes in the patient illustrated in Fig. 2 and 3. There is abnormal thinning during systole towards the apex.

and $\mathrm{J}$. Weiss using a computer-controlled video disc system (1978, personal communication) with manual identification of 16 points on endo- and epicardial boundaries. In principle, the limitation could also have been avoided by storing all the original information in semiconductor memory in the scanner, but this would have increased the cost very considerably. An additional advantage of the present method was its ability to make records of the original data on photographic paper rather than video tape.

These studies confirm the particular suitability of the isometric display or its variants for showing regional abnormalities in the timing and extent of left ventricular wall movement. They became apparent in a previous angiographic study (Gibson et al., 1976), and the similarity between these results and the present ones is close. Both show the synchronous pattern of wall movement in normal subjects, with systolic bulging and delayed relaxation as manifestations of local disease. The potential advantages of echocardiography, however, are considerable. In addition to being noninvasive, it is the method of choice for studying wall thickness or septal movement and we illustrate potential applications in these fields. As with angiography, simple inspection of images is not an adequate means of analysis, even for clinical purposes, and we present here a relatively cheap and simple adaptation, applicable to any phased array sector scanner, which allows a more satisfactory record to be made.

\section{References}

Gibson, D. G., and Brown, D. J. (1973). Measurement of instantaneous left ventricular dimension and filling rate in man, using echocardiography. British Heart fournal, 35, 1141-1149.

Gibson, D. G., Prewitt, T. A., and Brown, D. J. (1976) Analysis of left ventricular wall movement during isovolumic relaxation and its relation to coronary artery disease. British Heart fournal, 38, 1010-1019.

Hagan, A. D., Francis, G. S., Sahn, D. J., Karliner, J. S. Friedman, W. F., and O'Rourke, R. A. (1974). Ultrasound evaluation of systolic anterior septal motion in patients with right ventricular volume overload. Circulation, 50, 248-254.

Kisslo, J. A., Robertson, D., Gilbert, B. W., von Ramm, O., and Behar, V. S. (1977). A comparison of real-time two-dimensional echocardiography and cineangiography in detecting left ventricular asynergy. Circulation, 55, 134-141.

Weyman, A. E., Peskoe, S. M., Williams, E. S., Dillon, J. C., and Feigenbaum, H. (1976). Detection of left ventricular aneurysms by cross-sectional echocardiography. Circulation, 54, 936-943.

Requests for reprints to Dr D. G. Gibson, Cardiac Department, Brompton Hospital, Fulham Road, London SW3 6HP. 